\title{
Irregularity Measures for Metal-Organic Networks
}

\author{
Xuan Guo, ${ }^{1}$ Yu-Ming Chu $\left(\mathbb{D},{ }^{2,3}\right.$ Muhammad Khalid Hashmi, ${ }^{4}$ Abaid Ur Rehman Virk $\mathbb{D}^{\circ},{ }^{4}$ \\ and Jingjing $\mathrm{Li}^{5}$ \\ ${ }^{1}$ Automation School of Wuhan University of Technology, Wuhan 430070, China \\ ${ }^{2}$ Department of Mathematics, Huzhou University, Huzhou 313000, China \\ ${ }^{3}$ Hunan Provincial Key Laboratory of Mathematical Modeling and Analysis in Engineering, \\ Changsha University of Science \& Technology, Changsha 410114, China \\ ${ }^{4}$ Department of Mathematics, University of Lahore, Lahore, Pakistan \\ ${ }^{5}$ China Ship Development and Design Center, Wuhan 430064, China
}

Correspondence should be addressed to Yu-Ming Chu; chuyuming@zjhu.edu.cn

Received 30 April 2020; Accepted 16 June 2020; Published 1 August 2020

Guest Editor: Shaohui Wang

Copyright (c) 2020 Xuan Guo et al. This is an open access article distributed under the Creative Commons Attribution License, which permits unrestricted use, distribution, and reproduction in any medium, provided the original work is properly cited.

Topological index plays an important role in predicting physicochemical properties of a molecular structure. With the help of the topological index, we can associate a single number with a molecular graph. Drugs and other chemical compounds are frequently demonstrated as different polygonal shapes, trees, graphs, etc. In this paper, we will compute irregularity indices for metal-organic networks.

\section{Introduction}

Every planet is a blend of various kinds of parts, and every part has a significant commitment in the composition of the earth. The most significant parts in the earth are hydrogen, oxygen, and nitrogen. Subatomic hydrogen is one of the segments that has cordial situation and is a cutting edge wellspring of vitality $[1,2]$. As a gas, it can likewise be used in energy units to control engines. Among the various kinds of gases, hydrogen needs smell that makes any hole acknowledgment practically difficult to human creatures. The ongoing standards settled by the United States Vitality Sector put emphasis on the speed proficient of the gadget that can detect one percent by volume of drab and also scent-free atomic hydrogen in climate in less than sixty seconds in particular [3-6].

Jang et al. [7] presented an exceptionally quick hydrogen recognizing device comprising organic ligands and metals recognized with the assistance of palladium nanowires which can perceive hydrogen stages lower than 1 percent in just seven seconds. Moreover, other than distinguishing and detecting, the MONs appear exceptionally valuable with synthetic and physical properties, for example, grafting active groups [8], changing natural ligands [9], impregnating reasonable active materials [10], postsynthetic ligands, ion trade [11], and getting ready composites with useable substance [12].

Graph theory provides the interesting appliance in mathematical chemistry that is used to compute the various kinds of chemical compounds by means of graph theory and predict their various properties [13-19]. One of the most important tools in the chemical graph theory is a topological index, which is useful in predicting the chemical and physical properties of the underlying chemical compound, such as boiling point, strain energy, rigidity, heat of evaporation, and tension $[20,21]$. A graph having no loop or multiple edge is known as a simple graph. A molecular graph is a simple graph in which atoms and bounds are represented by the vertex and edge sets, respectively. The degree of the vertex is the number of edges attached with that vertex. These properties of various objects is of primary interest. Winner, in 1947, introduced the concept of the first topological index while finding the boiling point. In 1975, Gutman gave a remarkable identity [22] about Zagreb indices. Hence, these two indices are among the oldest degreebased descriptors, and their properties are extensively investigated. The mathematical formulae of these indices are 


$$
\begin{aligned}
& M_{1}(G)=\sum_{u v \in E(G)}\left(d_{u}+d_{v}\right), \\
& M_{2}(G)=\sum_{u v \in E(G)}\left(d_{u} \times d_{v}\right) .
\end{aligned}
$$

A topological index is known as the irregularity index [23] if the value of the topological index of the graph is greater than or equal to zero, and the topological index of the graph is equal to zero if and only if the graph is regular. The irregularity indices are given in Table 1. Most of the irregularity indices are from the family of degree-based topological indices and are used in quantitative structure activity relationship modeling.

For more about topological indices, one can read [24-31].

\section{Irregularity Indices for Metal- Organic Networks}

In this section, we discuss metal-organic networks by means of a graph. The unit cell of the metal-organic network is given in Figure 1. We give computational results of irregularity indices for two types of metal-organic networks $\mathrm{MON}_{1}(p)$ and $\mathrm{MON}_{2}(p)$ in the following two sections.

2.1. Irregularity Indices for Metal-Organic Network $\operatorname{MON}_{1}(p)$. This section is about irregularity indices of the metal-organic network $\mathrm{MON}_{1}(p)$. The molecular graph of the metal-organic network $\mathrm{MON}_{1}(p)$ for $p=2$ is given in Figure 2. We can observe from Figure 2 that there are four types of vertices present in the molecular graph of $\mathrm{MON}_{1}(p)$, i.e., 2, 3, 4, and 6. The cardinality of vertices 2,3 , 4 , and 6 is $30 p, 12,12 p-6$, and $6 p-6$, respectively. The cardinality of the vertex set of $\mathrm{MON}_{1}(p)$ is 48p, i.e., $\left|V\left(\mathrm{MON}_{1}(p)\right)\right|=48 p$. There are four different types of edges present in the molecular graph of $\operatorname{MON}_{1}(p)$, i.e., $\{2,3\},\{2,4\},\{2,6\}$, and $\{4,6\}$. Their cardinalities are 36, $36 p-12,24 p-24$, and $12 p-12$, respectively. The cardinality of the edge set of $\mathrm{MON}_{1}(p)$ is $72 p-12$, i.e., $\left|E\left(\mathrm{MON}_{1}(p)\right)\right|=72 p-12$.

The edge partition of the metal-organic network $\mathrm{MON}_{1}(p)$ is given in Table 2.

Theorem 1. Let $G$ be the metal-organic network $E\left(M_{1}(p)\right)$. The irregularity indices are
(1) $\operatorname{VAR}(G)=\left(8 p^{2}-5 p-1\right) / 4 p^{2}$
(2) $A L(G)=192 p-108$
(3) $\operatorname{IR} 1(G)=6\left(120 p^{2}-80 p-17\right) / p$
(4) $\operatorname{IR} 2(G)=(\sqrt{(864 p-456) / 48 p})-((72 p-12) / 24 p)$
(5) $\operatorname{IRF}(G)=576 p-444$
(6) $\operatorname{IRFW}(G)=48 p-37 / 2(36 p-19)$
(7) $\operatorname{IRA}(G)=48 p-2(\sqrt{6}+9 \sqrt{2}+4 \sqrt{3}) p-10 \sqrt{6}+$ $6 \sqrt{2}+8 \sqrt{3}$

TABLE 1: Definitions of irregularity indices.

\begin{tabular}{cc}
\hline Irregularity index & Mathematical form \\
\hline VAR & $\sum_{u \in V}\left(d_{u}-(2 m / n)\right)^{2}=\left(M_{1}(G) / n\right)-(2 m / n)^{2}$ \\
\hline AL & $\sum_{u v \in E(G)}\left|d_{u}-d_{v}\right|$ \\
\hline IR1 & $\sum_{u \in V}\left(d_{u}\right)^{3}-(2 m / n) \sum_{u \in V}\left(d_{u}\right)^{2}=F(G)-(2 m / n) M_{1}(G)$ \\
\hline IR2 & $\sqrt{\sum_{u v \in E(G)} d_{u} d_{v} / m}-(2 m / n)=\sqrt{M_{2}(G) / m}-(2 m / n)$ \\
\hline IRF & $\sum_{u v \in E(G)}\left(d_{u}-d_{v}\right)^{2}=F(G)-2 M_{2}(G)$ \\
\hline IRFW & $\operatorname{IRF}(G) / M_{2}(G)$ \\
\hline IRA & $\sum_{u v \in E(G)}\left(d_{u}^{-1 / 2}-d_{v}^{-1 / 2}\right)^{2}=n-2 R(G)$ \\
\hline IRB & $\sum_{u v \in E(G)}\left(d_{u}^{1 / 2}-d_{v}^{1 / 2}\right)^{2}=M_{1}(G)-2 R R(G)$ \\
\hline IRC & $\left(\sum_{u v \in E(G)} \sqrt{d_{u} d_{v}} / m\right)-(2 m / n)=(\operatorname{RR}(G) / m)-(2 m / n)$ \\
\hline IRDIF & $\sum_{u v \in E(G)}\left|\left(d_{u} / d_{v}\right)-\left(d_{v} / d_{u}\right)\right|=\sum_{i<j} m_{i, j}((j / i)-(i / j))$ \\
\hline IRL & $\sum_{u v \in E(G)}\left|\operatorname{lnd} d_{u}-\ln d_{v}\right|=\sum_{i<j} m_{i, j} \ln (j / i)$ \\
\hline IRLU & $\sum_{u v \in E(G)} \mid d_{u}-d_{v} / / m i n\left(d_{u}, d_{v}\right)=\sum_{i<j} m_{i, j} \ln ((j-i) / i)$ \\
\hline IRLF & $\sum_{u v \in E(G)}\left|d_{u}-d_{v}\right| / \sqrt{\left(d_{u} d_{v}\right)}=\sum_{i<j} m_{i, j}((j-i) / \sqrt{i j})$ \\
\hline IRLA & $2 \sum_{u v \in E(G)}\left|d_{u}-d_{v}\right| /\left(d_{u}+d_{v}\right)=2 \sum_{i<j} m_{i, j}((j-i) /(i+j))$ \\
\hline IRDI & $\sum_{u v \in E(G)}|\ln 1+| d_{u}-d_{v} \mid=\sum_{i<j} m_{i, j} \ln (i+j-1)$ \\
\hline IRGA & $\sum_{u v \in E(G)} \ln \left(\left(d_{u}+d_{v}\right) / 2 \sqrt{d_{u} d_{v}}\right) \sum_{i<j} m_{i, j}(i+j / 2 \sqrt{i j})$ \\
\hline
\end{tabular}

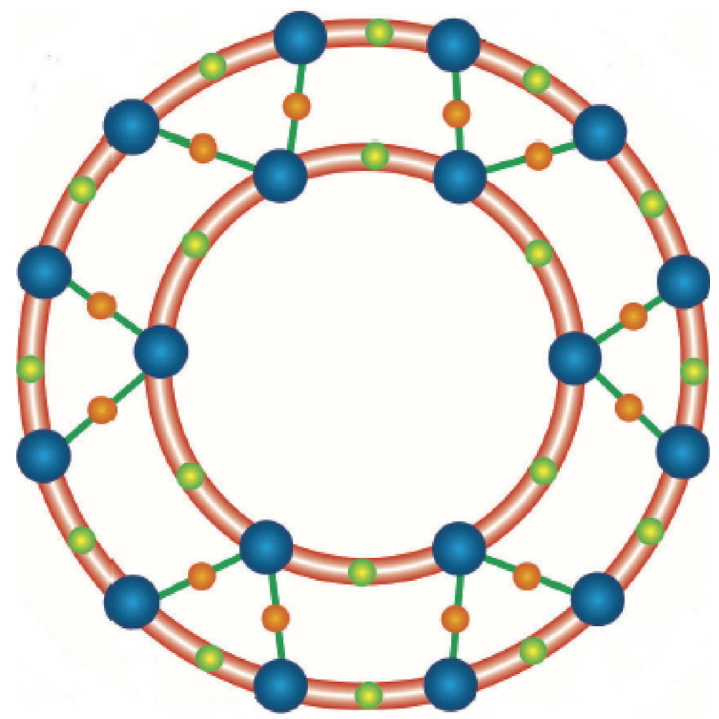

Figure 1: Basic metal-organic network.

(8) $\operatorname{IRB}(G)=528 p-204-(2(24 \sqrt{6}+72 \sqrt{2}+$ $48 \sqrt{3})) p-24 \sqrt{6}+48 \sqrt{2}+96 \sqrt{3}$

(9) $\operatorname{IRC}(G)=\left(4 \sqrt{6} p^{2}+2 \sqrt{6} p+12 \sqrt{2} p^{2}+8 \sqrt{3} p^{2}-\right.$ $\left.4 \sqrt{2} p-8 \sqrt{3} p-36 p^{2}+12 p-1\right) / 2 p(6 p-1)$

(10) $\operatorname{IRDIF}(G)=127.9980 p-61.9992$

(11) $\operatorname{IRL}(G)=56.1840 p-24.9516$

(12) $\operatorname{IRLU}(G)=90 p-48$

(13) $\operatorname{IRLF}(G)=58.0668 p-26.4012$

(14) $\operatorname{IRLA}(G)=52.7976 p-22.3992$

(15) $\operatorname{IRD} 1(G)=131.0064 p-42.4776$

(16) $\operatorname{IRGA}(G)=8.0172 p-5.8716$

Proof. Using definitions of irregularity indices given in Table 1 and edge partition given in Table 2, we have 


$$
\begin{aligned}
& \operatorname{VAR}(G)=\sum_{u \in V}\left(d_{u}-\frac{2 m}{n}\right)^{2}=\frac{M_{1}(G)}{n}-\left(\frac{2 m}{n}\right)^{2} \\
& =\left(\frac{528 p-204}{48 p}\right)-\left(\frac{2(72 p-12)}{48 p}\right)^{2} \\
& =\frac{\left(8 p^{2}-5 p-1\right)}{4 p^{2}} \\
& \operatorname{AL}(G)=\sum_{u v \in E(G)}\left|d_{u}-d_{v}\right| \\
& =|2-3|(36)+|2-4|(36 p-12)+|2-6|(24 p-1)+|4-6|(12 p-1) \\
& =192 p-108, \\
& \operatorname{IR} 1(G)=\sum_{u \in V} d_{u}^{3}-\frac{2 m}{n} \sum_{u \in V} d_{u}^{2}=F(G)-\left(\frac{2 m}{n}\right) M_{1}(G) \\
& =(2304 p-1356)-\frac{2(72 p-12)}{48 p}(528 p-204) \\
& =\frac{6\left(120 p^{2}-80 p-17\right)}{p} \\
& \operatorname{IR2}(G)=\sqrt{\frac{\sum_{u v \in E(G)} d_{u} d_{v}}{m}}-\frac{2 m}{n}=\sqrt{\frac{M_{2}(G)}{m}}-\frac{2 m}{n} \\
& =\sqrt{\frac{864 p-456}{72 p-12}}-\left(\frac{2(72 p-12)}{48 p}\right) \\
& =\sqrt{\frac{864 p-456}{48 p}}-\frac{72 p-12}{24 p}, \\
& \operatorname{IRF}(G)=\sum_{u v \in E(G)}\left(d_{u}-d_{v}\right)^{2} \\
& =(2-3)^{2}(36)+(2-4)^{2}(36 p-12)+(2-6)^{2}(24 p-1)+(4-6)^{2}(12 p-1) \\
& =576 p-444 \text {, } \\
& \operatorname{IRFW}(G)=\frac{\operatorname{IRF}(G)}{M_{2}(G)} \\
& =\frac{48 p-37}{2(36 p-19)} \\
& \operatorname{IRA}(G)=\sum_{u v \in E(G)}\left(d_{u}^{-1 / 2}-d_{v}^{-1 / 2}\right)^{2}=n-2 R(G) \\
& =(48 p)-2((9 \sqrt{2}+\sqrt{3}+\sqrt{6}) p-(3 \sqrt{2}+4 \sqrt{3}-5 \sqrt{6})) \\
& =48 p-(2(\sqrt{6}+9 \sqrt{2}+4 \sqrt{3})) p-10 \sqrt{6}+6 \sqrt{2}+8 \sqrt{3} \text {, } \\
& \operatorname{IRB}(G)=\sum_{u v \in E(G)}\left(d_{u}^{1 / 2}-d_{v}^{1 / 2}\right)^{2}=M_{1}\left(D_{n} P_{n}\right)-2 \operatorname{RR}(G) \\
& =(528 p-204)-2((72 \sqrt{2}+48 \sqrt{3}+24 \sqrt{6}) p-(24 \sqrt{2}+48 \sqrt{3}-12 \sqrt{6}))
\end{aligned}
$$




$$
\begin{aligned}
& =528 p-204-(2(24 \sqrt{6}+72 \sqrt{2}+48 \sqrt{3})) p-24 \sqrt{6}+48 \sqrt{2}+96 \sqrt{3} \text {, } \\
& \operatorname{IRC}(G)=\frac{\sum_{u v \in E(G)} \sqrt{d_{u} d_{v}}}{m}-\frac{2 m}{n}=\frac{\operatorname{RR}(G)}{m}-\frac{2 m}{n} \\
& =\frac{(72 \sqrt{2}+48 \sqrt{3}+24 \sqrt{6}) p-(24 \sqrt{2}+48 \sqrt{3}-12 \sqrt{6})}{72 p-12} \frac{2(72 p-12)}{48 p} \\
& =\frac{4 \sqrt{6} p^{2}+2 \sqrt{6} p+12 \sqrt{2} p^{2}+8 \sqrt{3} p^{2}-4 \sqrt{2} p-8 \sqrt{3} p-36 p^{2}+12 p-1}{2 p(6 p-1)}, \\
& \operatorname{IRDIF}(G)=\sum_{u v \in E(G)}\left|\frac{d_{u}}{d_{v}}-\frac{d_{v}}{d_{u}}\right| \\
& =\left|\frac{2}{3}-\frac{3}{2}\right|(36)+\left|\frac{2}{4}-\frac{4}{2}\right|(36 p-12)+\left|\frac{2}{6}-\frac{6}{2}\right|(24 p-1)+\left|\frac{4}{6}-\frac{6}{4}\right|(12 p-1) \\
& =127.9980 p-61.9992 \text {, } \\
& \operatorname{IRL}(G)=\sum_{u v \in E(G)}\left|\operatorname{lnd} \mathrm{d}_{u}-\ln \mathrm{d}_{v}\right| \\
& =|\ln 2-\ln 3|(36)+|\ln 2-\ln 4|(36 p-12) \\
& +|\ln 2-\ln 6|(24 p-1)+|\ln 4-\ln 6|(12 p-1) \\
& =56.1840 p-24.9516 \text {, } \\
& \operatorname{IRLU}(G)=\sum_{u v \in E(G)} \frac{\left|d_{u}-d_{v}\right|}{\min \left(d_{u}, d_{v}\right)} \\
& =\frac{|2-3|}{2}(36)+\frac{|2-4|}{2}(36 p-12)+\frac{|2-6|}{2}(24 p-1)+\frac{|4-6|}{4}(12 p-1) \\
& 90 p-48 \\
& \operatorname{IRLF}(G)=\sum_{u v \in E(G)} \frac{\left|d_{u}-d_{v}\right|}{\sqrt{d_{u} \cdot d_{v}}} \\
& =\frac{|2-3|}{\sqrt{6}}(36)+\frac{|2-4|}{\sqrt{8}}(36 p-12)+\frac{|2-6|}{\sqrt{12}}(24 p-1)+\frac{|4-6|}{\sqrt{24}}(12 p-1) \\
& =58.0668 p-26.4012 \text {, } \\
& \operatorname{IRLA}(G)=\sum_{u v \in E(G)} 2 \frac{\left|d_{u}-d_{v}\right|}{\left(d_{u}+d_{v}\right)} \\
& =2 \frac{|2-3|}{5}(36)+2 \frac{|2-4|}{6}(36 p-12)+2 \frac{|2-6|}{8}(24 p-1)+2 \frac{|4-6|}{10}(12 p-1) \\
& =52.7976 p-22.3992 \text {, } \\
& \operatorname{IRD} 1(G)=\sum_{u v \in E(G)} \ln \left\{1+\left|d_{u}-d_{v}\right|\right\} \\
& =\ln \{1+|2-3|\}(36)+\ln \{1+|2-4|\}(36 p-12)+\ln \{1+|2-6|\}(24 p-1)+\ln \{1+|4-6|\}(12 p-1) \\
& =131.0064 p-42.4776 \text {, } \\
& \operatorname{IRGA}(G)=\sum_{u v \in E(G)} \ln \left(\frac{d_{u}+d_{v}}{2 \sqrt{d_{u} d_{v}}}\right) \\
& =\ln \left(\frac{2+3}{2 \sqrt{2 \times 3}}\right)(36)+\ln \left(\frac{2+4}{2 \sqrt{2 \times 4}}\right)(36 p-1)+\ln \left(\frac{2+6}{2 \sqrt{2 \times 6}}\right)(24 p-1)+\ln \left(\frac{4+6}{2 \sqrt{4 \times 6}}\right)(12 p-1) \\
& =8.0172 p-5.8716 \text {. }
\end{aligned}
$$

2.2. Irregularity Indices for Metal-Organic Network $M O N_{2}(p)$. In this section, we will compute irregularity indices for the metal-organic network $\mathrm{MON}_{2}(p)$. The molecular graph of the metal-organic network $\mathrm{MON}_{2}(p)$ for $p=2$ is given in Figure 3. It is clear from Figure 3 that there are three types of vertices in the molecular graph of 


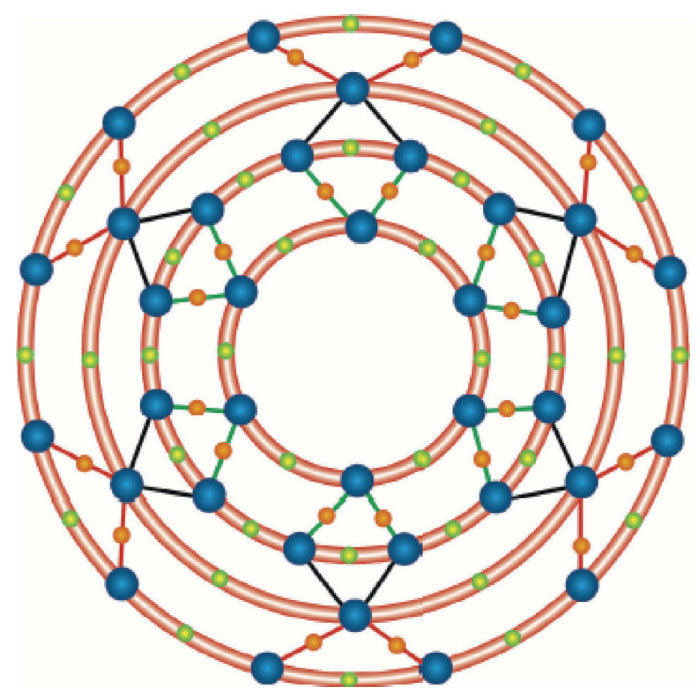

Figure 2: $\mathrm{MON}_{1}(p)$ for $p=2$.

TABle 2: Partition of $E\left(\mathrm{MON}_{1}(p)\right)$.

\begin{tabular}{lc}
\hline$\left(d_{u}, d_{v}\right)$ & Frequency \\
\hline$(2,3)$ & 36 \\
$(2,4)$ & $12(3 p-1)$ \\
$(2,6)$ & $24(p-1)$ \\
$(4,6)$ & $12(p-1)$ \\
\hline
\end{tabular}

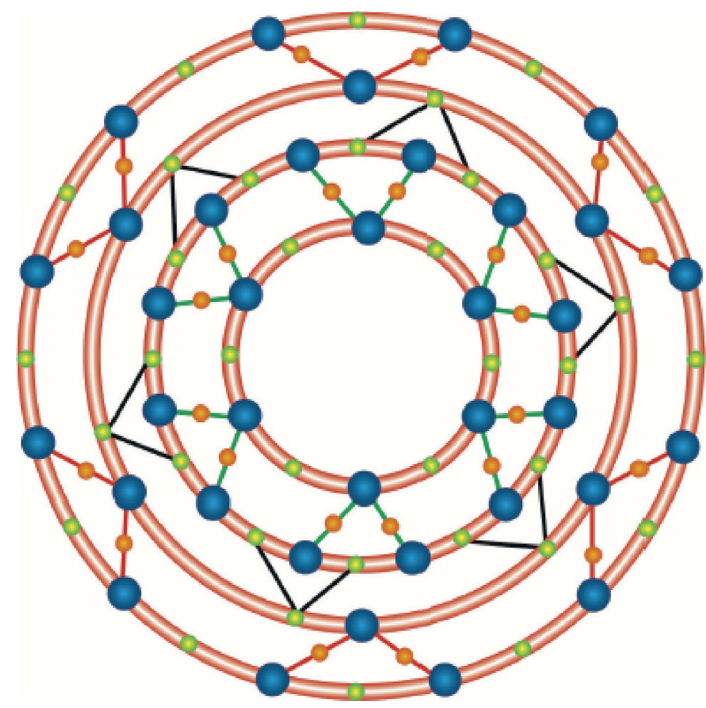

Figure 3: $\mathrm{MON}_{2}(p)$ for $p=2$.

$\mathrm{MON}_{2}(p)$, i.e., 2, 3, and 4. The cardinality of vertices 2, 3, and 4 is $12 p+18,24 p-12$, and $12 p-6$, respectively. The cardinality of the vertex set of $\mathrm{MON}_{2}(p)$ is $48 p$, i.e., $\left|V\left(\mathrm{MON}_{2}(p)\right)\right|=48 p$. There are five different types of edges present in the molecular graph of $\operatorname{MON}_{2}(p)$, i.e., $\{2,3\}$, $\{2,4\},\{3,3\},\{3,4\}$, and $\{4,4\}$. Their cardinalities are $12 p+24,12 p+12,24 p-24,12 p-12$, and $12 p-12$, respectively. The cardinality of the edge set of $\mathrm{MON}_{2}(p)$ is $72 p-12$, i.e., $\left|E\left(\mathrm{MON}_{1}(p)\right)\right|=72 p-12$.

The edge partition of the metal-organic network $\mathrm{MON}_{2}(p)$ is given in Table 3 .
Table 3: Partition of $E\left(\operatorname{MON}_{2}(p)\right)$.

\begin{tabular}{lc}
\hline$\left(d_{u}, d_{v}\right)$ & Frequency \\
\hline$(2,3)$ & $12(p+2)$ \\
$(2,4)$ & $12(p+1)$ \\
$(3,3)$ & $24(p-1)$ \\
$(3,4)$ & $12(p-1)$ \\
$(4,4)$ & $12(p-1)$ \\
\hline
\end{tabular}

Theorem 2. $E\left(M_{2}(p)\right)$ Let $G$ be the metal-organic network $\mathrm{MON}_{2}(p)$. The irregularity indices are

(1) $\operatorname{VAR}(G)=\left(2 p^{2}+p-1\right) / 4 p^{2}$

(2) $A L(G)=48 p+36$

(3) $I R 1(G)=6\left(24 p^{2}+10 p-11\right) / p$

(4) $\operatorname{IR} 2(G)=\sqrt{(720 p-312) / 48 p}-((72 p-12) / 24 p)$

(5) $\operatorname{IRF}(G)=72 p+60$

(6) $\operatorname{IRFW}(G)=(72 p+60) /(720 p-312)$

(7) $\operatorname{IRA}(G)=32 p+16-2(2 \sqrt{6}+4 \sqrt{3}+3 \sqrt{2}) p-$ $8 \sqrt{6}-6 \sqrt{2}+8 \sqrt{3}$

(8) $\operatorname{IRB}(G)=216 p+108-(2(12 \sqrt{6}+24 \sqrt{2}+$ $24 \sqrt{3})) p-48 \sqrt{6}-48 \sqrt{2}+48 \sqrt{3}$

(9) $\operatorname{IRC}(G)=\left(2 \sqrt{6} p^{2}+4 \sqrt{6} p+4 \sqrt{3} p^{2}+4 \sqrt{2} p^{2}-\right.$ $\left.4 \sqrt{3} p+4 \sqrt{2} p-16 p^{2}-8 p-1\right) / 2 p(6 p-1)$

(10) $\operatorname{IRDIF}(G)=34.9992 p+30.9996$

(11) $\operatorname{IRL}(G)=16.8660 p+14.3664$

(12) $\operatorname{IRLU}(G)=21.9996 p+20.0004$

(13) $\operatorname{IRLF}(G)=16.8468 p+14.8188$

(14) $\operatorname{IRLA}(G)=16.2276 p+14.1708$

(15) $\operatorname{IRD} 1(G)=29.8176 p+21.5004$

(16) $\operatorname{IRGA}(G)=1.0740 p+1.0716$

Proof. Using definitions of irregularity indices given in Table 1 and edge partition given in Table 3, we have 


$$
\begin{aligned}
& \operatorname{VAR}(G)=\sum_{u \varepsilon V}\left(d_{u}-\frac{2 m}{n}\right)^{2}=\frac{M_{1}(G)}{n}-\left(\frac{2 m}{n}\right)^{2} \\
& =\left(\frac{456 p-132}{48 p}\right)-\left(\frac{2(72 p-12)}{48 p}\right)^{2} \\
& =\frac{\left(2 p^{2}+p-1\right)}{4 p^{2}} \\
& \operatorname{AL}(G)=\sum_{u v \in E(G)}\left|d_{u}-d_{v}\right| \\
& =|2-3|(12 p+24)+|2-4|(12 p+12)+|3-3|(24 p-24)+|3-4|(12 p-12)+|4-4|(12 p-12) \\
& =48 p+36 \text {, } \\
& \operatorname{IR} 1(G)=\sum_{u \in V} d_{u}^{3}-\frac{2 m}{n} \sum_{u \in V} d_{u}^{2}=F(G)-\left(\frac{2 m}{n}\right) M_{1}(G) \\
& =(1512 p-564)-\frac{2(72 p-12)}{48 p}(456 p-132) \\
& =\frac{6\left(24 p^{2}+10 p-11\right)}{p} \\
& \operatorname{IR2}(G)=\sqrt{\frac{\sum_{u v \in E(G)} d_{u} d_{v}}{m}}-\frac{2 m}{n}=\sqrt{\frac{M_{2}(G)}{m}}-\frac{2 m}{n} \\
& =\sqrt{\frac{720 p-312}{72 p-12}}-\left(\frac{(272 p-12)}{48 p}\right) \\
& =\sqrt{\frac{720 p-312}{48 p}-\frac{72 p-12}{24 p}}, \\
& \operatorname{IRF}(G)=\sum_{u v \in E(G)}\left(d_{u}-d_{v}\right)^{2} \\
& =(2-3)^{2}(12 p+24)+(2-4)^{2}(12 p+12)+(3-3)^{2}(24 p-24)+(3-4)^{2}(12 p-12)+(4-4)^{2}(12 p-12) \\
& =72 p+60 \text {, } \\
& \operatorname{IRFW}(G)=\frac{\operatorname{IRF}(G)}{M_{2}(G)} \\
& =\frac{72 p+60}{720 p-312} \\
& \begin{aligned}
\operatorname{IRA}(G) & =\sum_{u v \in E(G)}\left(d_{u}^{-1 / 2}-d_{v}^{-1 / 2}\right)^{2}=n-2 R(G)=(48 p)-2(8 p-8+(3 \sqrt{2}+4 \sqrt{3}+2 \sqrt{6}) p+(3 \sqrt{2}-4 \sqrt{3}+4 \sqrt{6})) \\
& =32 p+16-2(2 \sqrt{6}+4 \sqrt{3}+3 \sqrt{2}) p-8 \sqrt{6}-6 \sqrt{2}+8 \sqrt{3}
\end{aligned} \\
& \operatorname{IRB}(G)=\sum_{u v \varepsilon E(G)}\left(d_{u}^{1 / 2}-d_{v}^{1 / 2}\right)^{2}=M_{1}(G)-2 \operatorname{RR}(G) \\
& =(456 p-132)-2(120 p-120+(24 \sqrt{2}+24 \sqrt{3}+12 \sqrt{6}) p+(24 \sqrt{6}-24 \sqrt{3}+24 \sqrt{2})) \\
& =216 p+108-(2(12 \sqrt{6}+24 \sqrt{2}+24 \sqrt{3})) p-48 \sqrt{6}-48 \sqrt{2}+48 \sqrt{3} \text {, }
\end{aligned}
$$




$$
\begin{aligned}
& \operatorname{IRC}(G)=\frac{\sum_{u v \in E(G)} \sqrt{d_{u} d_{v}}}{m}-\frac{2 m}{n}=\frac{\operatorname{RR}(G)}{m}-\frac{2 m}{n} \\
& =\frac{120 p-120+(24 \sqrt{2}+24 \sqrt{3}+12 \sqrt{6}) p+(24 \sqrt{6}-24 \sqrt{3}+24 \sqrt{2})}{72 p-12} \frac{2(72 p-12)}{48 p} \\
& =\frac{2 \sqrt{6} p^{2}+4 \sqrt{6} p+4 \sqrt{3} p^{2}+4 \sqrt{2} p^{2}-4 \sqrt{3} p+4 \sqrt{2} p-16 p^{2}-8 p-1}{2 p(6 p-1)}, \\
& \operatorname{IRDIF}(G)=\sum_{u v \in E(G)}\left|\frac{d_{u}}{d_{v}}-\frac{d_{v}}{d_{u}}\right| \\
& =\left|\frac{2}{3}-\frac{3}{2}\right|(12 p+24)+\left|\frac{2}{4}-\frac{4}{2}\right|(12 p+12)+\left|\frac{3}{3}-\frac{3}{3}\right|(24 p-24)+\left|\frac{3}{4}-\frac{4}{3}\right|(12 p-12)+\left|\frac{4}{4}-\frac{4}{4}\right|(12 p-12), \\
& =34.9992 p+30.9996 \text {, } \\
& \operatorname{IRL}(G)=\sum_{u v \in E(G)}\left|\operatorname{lnd} \mathrm{d}_{u}-\operatorname{lnd}_{v}\right| \\
& =|\ln 2-\ln 3|(12 p+24)+|\ln 2-\ln 4|(12 p+12)+|\ln 3-\ln 3|(24 p-24)+|\ln 3-\ln 4|(12 p-12) \\
& +|\ln 4-\ln 4|(12 p-12) \\
& =16.8660 p+14.3664 \text {, } \\
& \operatorname{IRLU}(G)=\sum_{u v \in E(G)} \frac{\left|d_{u}-d_{v}\right|}{\min \left(d_{u}, d_{v}\right)} \\
& =\frac{|2-3|}{2}(12 p+24)+\frac{|2-4|}{2}(12 p+24)+\frac{|3-3|}{3}(24 p-24)+\frac{|3-4|}{3}(12 p-12)+\frac{|4-4|}{4}(12 p-12) \\
& =21.9996 p+20.0004 \\
& \operatorname{IRLF}(G)=\sum_{(u v \in E G)} \frac{\left|d_{u}-d_{v}\right|}{\sqrt{d_{u} \cdot d_{v}}} \\
& =\frac{|2-3|}{\sqrt{6}}(12 p+24)+\frac{|2-4|}{\sqrt{8}}(12 p+12)+\frac{|3-3|}{\sqrt{9}}(24 p-24)+\frac{|3-4|}{\sqrt{12}}(12 p-12)+\frac{|4-4|}{\sqrt{16}}(12 p-12) \\
& =16.8468 p+14.8188 \text {, } \\
& \operatorname{IRLA}(G)=\sum_{u v \in E(G)} 2 \frac{\left|d_{u}-d_{v}\right|}{\left(d_{u}+d_{v}\right)} \\
& =2 \frac{|2-3|}{5}(12 p+24)+2 \frac{|2-4|}{6}(12 p+12)+2 \frac{|3-3|}{6}(24 p-24)+2 \frac{|3-4|}{7}(12 p-12)+2 \frac{|4-4|}{8}(12 p-12) \\
& =16.2276 p+14.1708 \text {, } \\
& \operatorname{IRD} 1(G)=\sum_{u v \in E(G)} \ln \left\{1+\left|d_{u}-d_{v}\right|\right\} \\
& =\ln \{1+|2-3|\}(12 p+24)+\ln \{1+|2-4|\}(12 p+12)+\ln \{1+|3-3|\}(24 p-24)+\ln \{1+|3-4|\}(12 p-12) \\
& +\ln \{1+|4-4|\}(12 p-12) \\
& =29.8176 p+21.5004 \text {, } \\
& \operatorname{IRGA}(G)=\sum_{u v \in E(G)} \ln \left(\frac{d_{u}+d_{v}}{2 \sqrt{d_{u} d_{v}}}\right) \\
& =\ln \left(\frac{2+3}{2 \sqrt{2 \times 3}}\right)(12 p+24)+\ln \left(\frac{2+4}{2 \sqrt{2 \times 4}}\right)(12 p+12)+\ln \left(\frac{3+3}{2 \sqrt{3 \times 3}}\right)(24 p-24)+\ln \left(\frac{3+4}{2 \sqrt{3 \times 4}}\right)(12 p-12) \\
& +\ln \left(\frac{4+4}{2 \sqrt{4 \times 4}}\right)(12 p-12) \\
& =1.0740 p+1.0716 \text {. }
\end{aligned}
$$




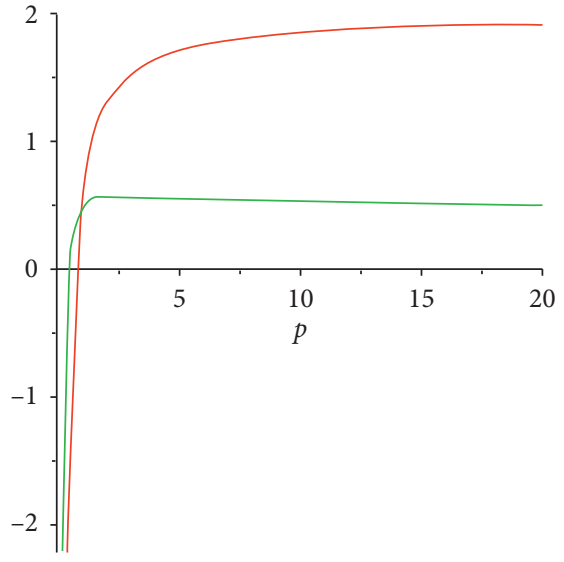

(a)

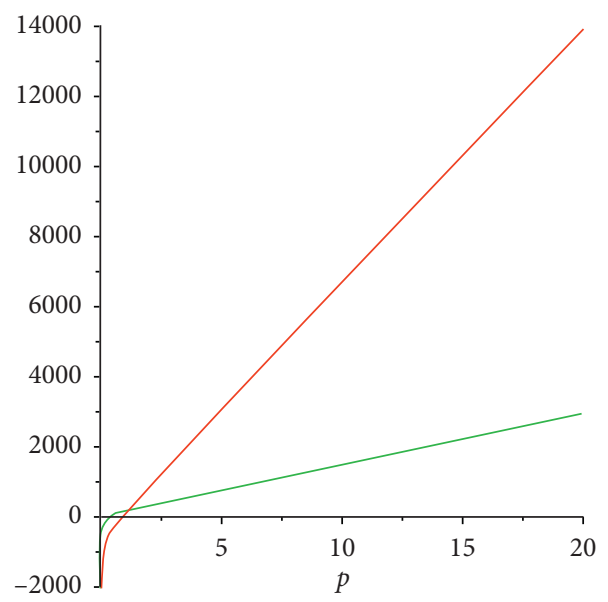

(c)

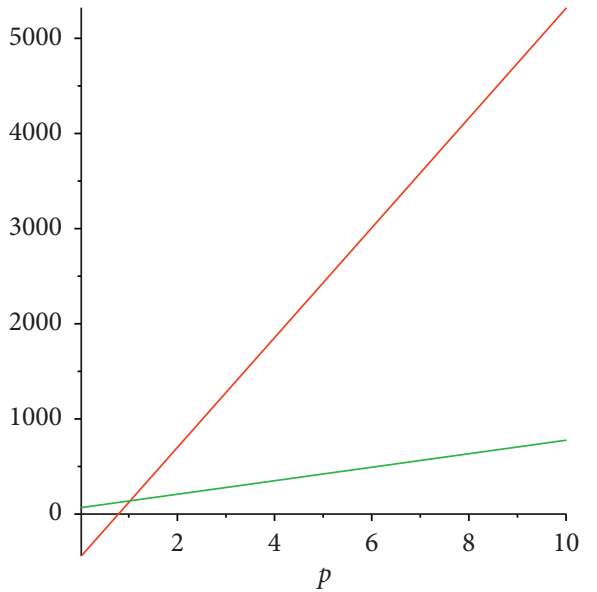

(e)

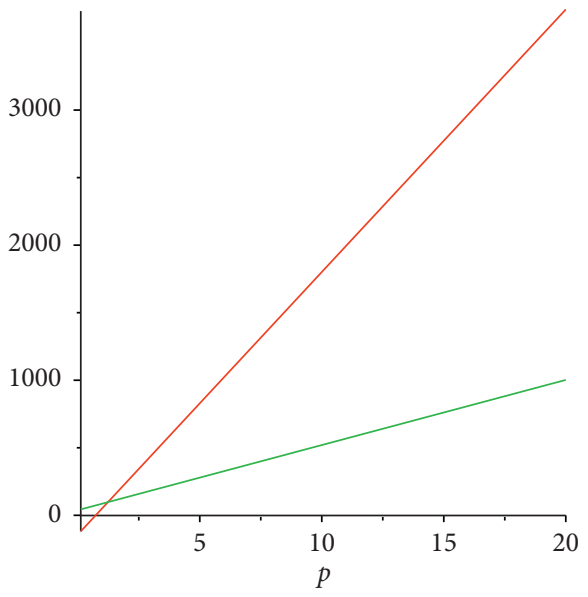

(b)

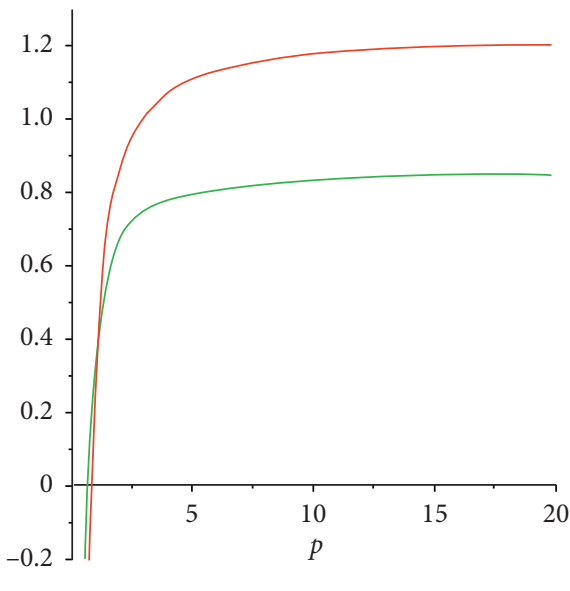

(d)

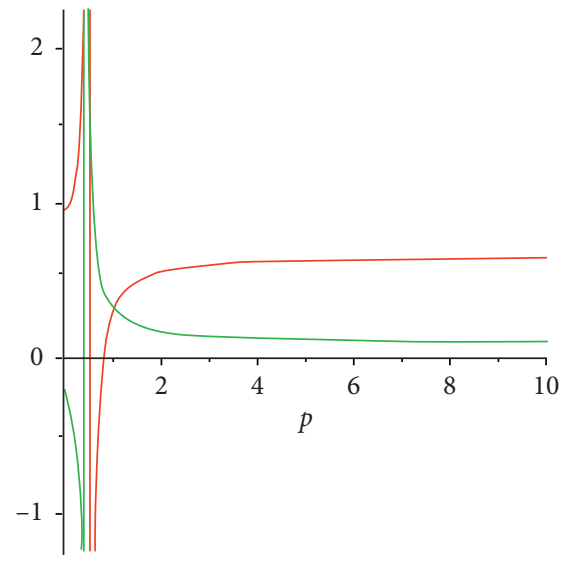

(f)

Figure 4: Continued. 


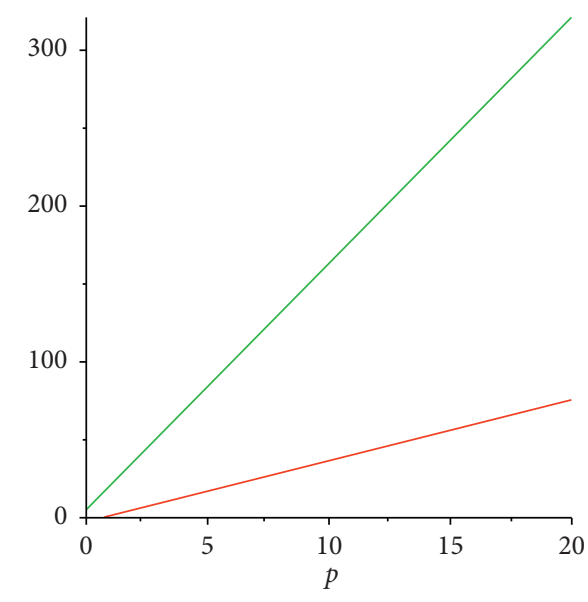

(g)

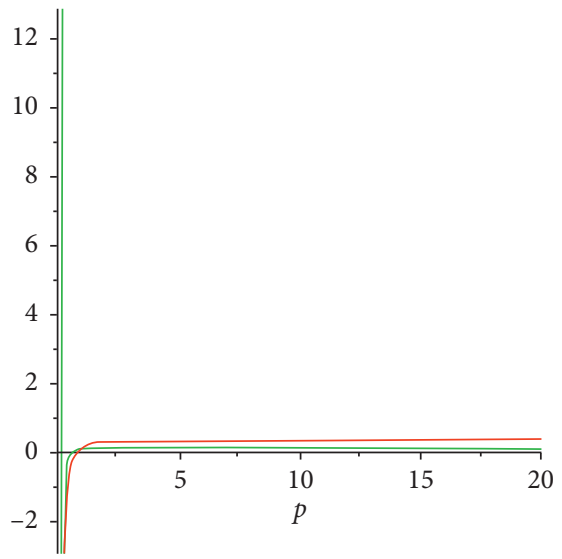

(i)

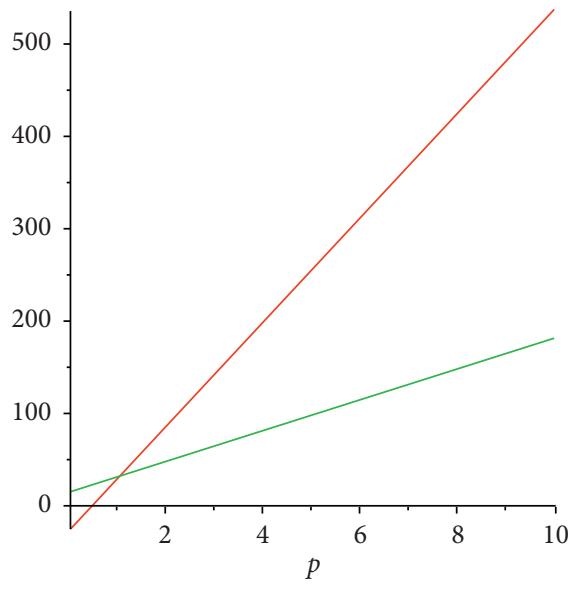

(k)

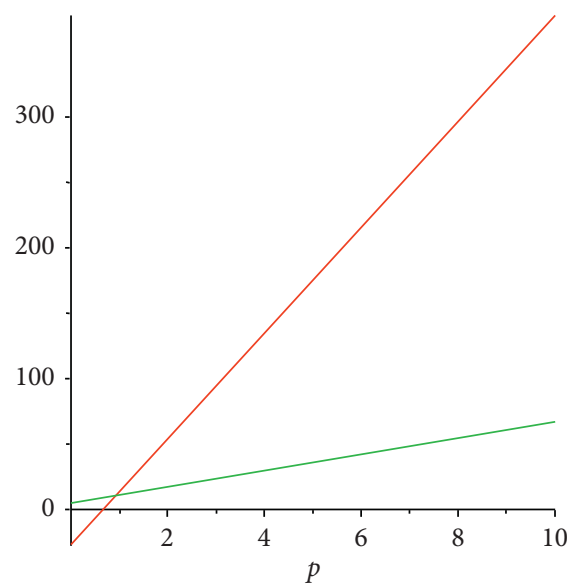

(h)

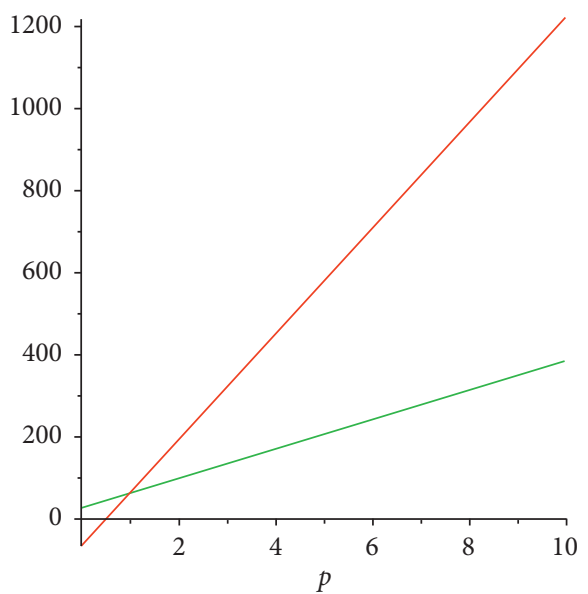

(j)

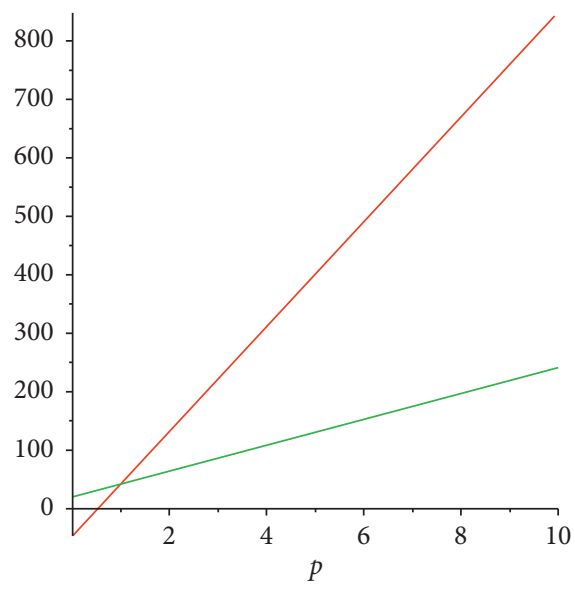

(l)

Figure 4: Continued. 


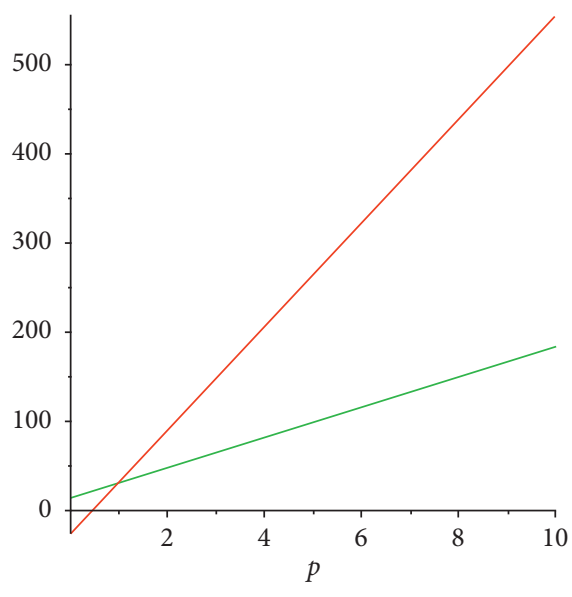

(m)

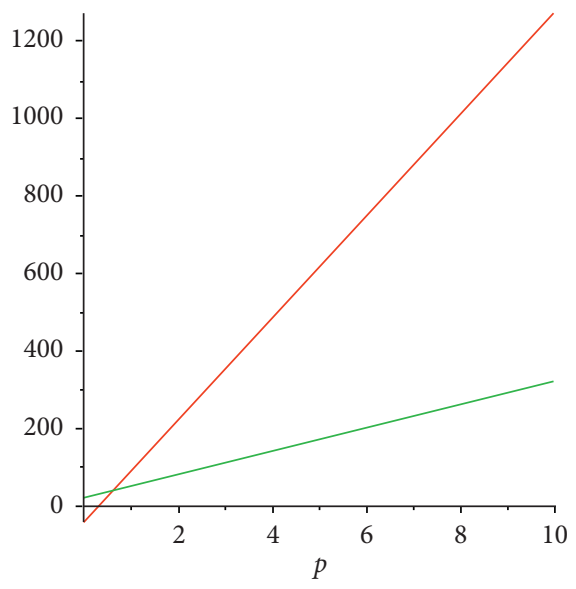

(o)

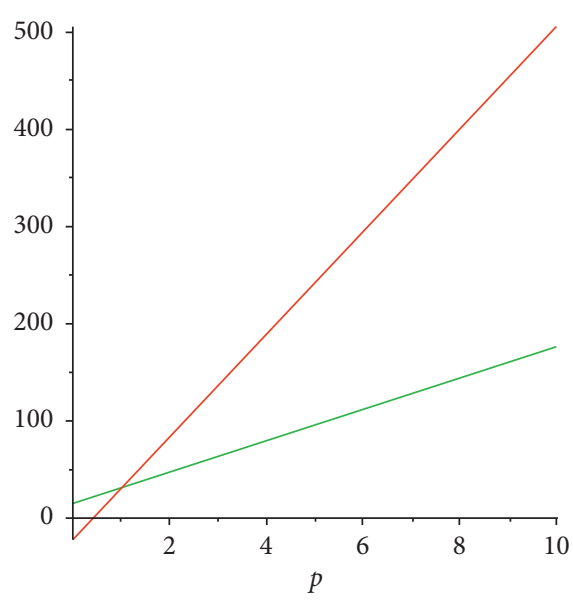

(n)

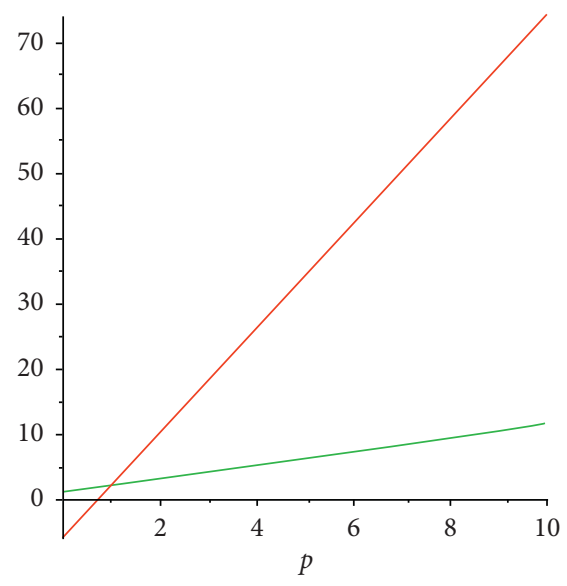

(p)

FIgURE 4: Graphical comparison of irregularity indices. (a) VAR. (b) AL. (c) IR1. (d) IR2. (e) IRF. (f) IRFW. (g) IRA. (h) IRB. (i) IRC. (j) IRDIF. (k) IRL. (l) IRLU. (m) IRLF. (n) IRLA. (o) IRD1. (p) IRGA.

\section{Graphical Comparison}

In this section, we give the graphical comparison of results of the metal-organic networks $\mathrm{MON}_{1}(p)$ and $\mathrm{MON}_{2}(p)$. In Figure 4, the red color is fixed for $\mathrm{MON}_{1}(p)$ and the green color is fixed for $\mathrm{MON}_{2}(p)$.

\section{Conclusion}

Topological indices associate a single number with a chemical structure. In quantitative structure activity relationship, knowledge of topological indices plays an important role. In this article, we computed sixteen irregularity indices for metalorganic networks $\mathrm{MON}_{1}(p)$ and $\mathrm{MON}_{2}(p)$. Most of the calculated topological indices depend on degree-based indices. In the field of chemical graph theory, molecular topology, and mathematical chemistry, a degree-based index also known as a connectivity index is a type of a molecular descriptor that is calculated based on the molecular graph of a chemical compound. Topological indices are numerical parameters of a graph which characterize its topology and are usually graph invariant. Topological indices are used, for example, in the development of quantitative structure activity relationships (QSARs) in which the biological activity or other properties of molecules are correlated with their chemical structure. Our results are helpful in drug delivery and computer engineering.

\section{Data Availability}

All data required for this research are included within this paper.

\section{Conflicts of Interest}

The authors do not have any conflicts of interest.

\section{Authors' Contributions}

All authors contributed equally in this paper.

\section{Acknowledgments}

The research was supported by the National Natural Science Foundation of China (Grant nos. 11971142, 11871202, $61673169,11701176,11626101$, and 11601485). 


\section{References}

[1] C. Petit and T. J. Bandosz, "MOF-graphite oxide composites: combining the uniqueness of graphene layers and metalorganic frameworks," Advanced Materials, vol. 21, no. 46, pp. 4753-4757, 2009.

[2] S. J. Yang, J. Y. Choi, H. K. Chae, J. H. Cho, K. S. Nahm, and C. R. Park, "Preparation and enhanced hydrostability and hydrogen storage capacity of CNT@MOF-5 hybrid composite," Chemistry of Materials, vol. 21, no. 9, pp. 1893-1897, 2009.

[3] X. Li and J. Zheng, "Extremal chemical trees with minimum or maximum general Randic index," MATCH Communications in Mathematical and in Computer Chemistry, vol. 55, no. 2, pp. 381-390, 2006.

[4] M. C. Wasson, J. Lyu, T. Islamoglu, and O. K. Farha, "Linker competition within a metal-organic framework for topological insights," Inorganic Chemistry, vol. 58, no. 2, pp. 15131517, 2018.

[5] I. Gutman, A. M. Naji, and N. D. Soner, "On leap Zagreb indices of graphs," Communication in Combinatorics and Optimization, vol. 2, no. 2, pp. 99-117, 2017.

[6] J. Liu and Y. Lu, "Accelerated color change of gold nanoparticles assembled by DNAzymes for simple and fast colorimetric $\mathrm{Pb}^{2+}$ Detection," Journal of the American Chemical Society, vol. 126, no. 39, pp. 12298-12305, 2004.

[7] J.-S. Jang, S. Qiao, S.-J. Choi et al., "Hollow Pd-Ag composite nanowires for fast responding and transparent hydrogen sensors," ACS Applied Materials \& Interfaces, vol. 9, no. 45, pp. 39464-39474, 2017.

[8] Y. K. Hwang, D.-Y. Hong, J.-S. Chang et al., “Amine grafting on coordinatively unsaturated metal centers of MOFs: consequences for catalysis and metal encapsulation," Angewandte Chemie International Edition, vol. 47, no. 22, pp. 4144-4148, 2008.

[9] D. Bradshaw, A. Garai, and J. Huo, "Metal-organic framework growth at functional interfaces: thin films and composites for diverse applications," Chemical Society Reviews, vol. 41, no. 6 , pp. 2344-2381, 2012.

[10] A. W. Thornton, K. M. Nairn, J. M. Hill, A. J. Hill, and M. R. Hill, "Metal-organic frameworks impregnated with magnesium-decorated fullerenes for methane and hydrogen storage," Journal of the American Chemical Society, vol. 131, no. 30, pp. 10662-10669, 2009.

[11] M. Azari and A. Iranmanesh, "Generalized zagreb index of graphs," Studia Universitatis Babes-Bolyai, Chemia, vol. 56, no. 3, pp. 59-70, 2011.

[12] A. Vasilyev, "Upper and lower bounds of symmetric division deg index," Iranian Journal of Mathematical Chemistry, vol. 5, no. 2, pp. 91-98, 2014.

[13] N. Ali, M. A. Umar, and A. Tabassum, "Super $(a, d)-C 3-$ antimagicness of a corona graph," Open Journal of Mathematical Sciences, vol. 2, no. 1, pp. 371-378, 2018.

[14] M. A. Umar, M. A. Javed, M. Hussain, and B. R. Ali, "Super ( $a$, d)-C 4-antimagicness of book graphs," Open Journal of Mathematical Sciences, vol. 2, no. 1, pp. 115-121, 2018.

[15] M. A. Umar, "Cyclic-antimagic construction of ladders," Engineering and Applied Science Letters, vol. 2, no. 2, pp. 43-47, 2019.

[16] M. A. Umar, N. Ali, N. Ali, A. Tabassum, and B. R. Ali, "Book graphs are cycle antimagic," Open Journal of Mathematical Sciences, vol. 3, no. 1, pp. 184-190, 2019.

[17] A. Tabassum, M. A. Lingua, M. A. Umar, M. Perveen, and A. Raheem, "Antimagicness of subdivided fans," Open Journal of Mathematical Sciences, vol. 4, no. 1, pp. 18-22, 2020.
[18] F. Aslam, Z. Zahid, Z. Zahid, and S. Zafar, "3-total edge mean cordial labeling of some standard graphs," Open Journal of Mathematical Sciences, vol. 3(2019), no. 1, pp. 129-138, 2019.

[19] F. Asif, Z. Zahid, and S. Zafar, "Leap Zagreb and leap hyperZagreb indices of Jahangir and Jahangir derived graphs," Engineering and Applied Science Letter, vol. 3, no. 2, pp. 1-8, 2020.

[20] F. M. Brckler, T. Dolic, A. Graovac, and I. Gutman, "On a class of distance-based molecular structure descriptors," Chemical Physics Letters, vol. 503, no. 4-6, pp. 336-338, 2011.

[21] H. Gonzalez-Diaz, S. Vilar, L. Santana, and E. Uriarte, "Medicinal chemistry and bioinformatics-current trends in drugs discovery with networks topological indices," Current Topics in Medicinal Chemistry, vol. 7, no. 10, pp. 1015-1029, 2007.

[22] H. Hosoya, K. Hosoi, and I. Gutman, "A topological index for the totalp-electron energy," Theoretica Chimica Acta, vol. 38, no. 1, 1975.

[23] T. Réti, R. Sharafdini, A. Dregelyi-Kiss, and H. Haghbin, "Graph irregularity indices used as molecular descriptors in QSPR studies," MATCH Communications in Mathematical and in Computer Chemistry, vol. 79, pp. 509-524, 2018.

[24] E. Deutsch and S. Klavzar, "S. M-Polynomial and degreebased topological indices," Iranian Journal of Mathematical Chemistry, vol. 6, pp. 93-102, 2015.

[25] M. Ajmal, W. Nazeer, M. Munir, S. M. Kang, and C. Y. Jung, "The M-polynomials and topological indices of generalized prism network," International Journal of Mathematical Analysis, vol. 11, no. 6, pp. 293-303, 2017.

[26] M. Munir, W. Nazeer, Z. Shahzadi, and S. Kang, "Some invariants of circulant graphs," Symmetry, vol. 8, no. 11, p. 134, 2016.

[27] H. Wiener, "Structural determination of paraffin boiling points," Journal of the American Chemical Society, vol. 69, no. 1, pp. 17-20, 1947.

[28] A. A. Dobrynin, R. Entringer, and I. Gutman, "Wiener index of trees: theory and applications," Acta Applicandae Mathematicae, vol. 66, no. 3, pp. 211-249, 2001.

[29] I. Gutman and O. E. Polansky, Mathematical Concepts in Organic Chemistry, Springer Science \& Business Media, Berlin, Germany, 2012.

[30] M. Randic, "Characterization of molecular branching," Journal of the American Chemical Society, vol. 97, no. 23, pp. 6609-6615, 1975.

[31] B. Bollobás, P. Erdös, and A. Sarkar, "Graphs of extremal weights,” Ars Combinatoria, vol. 50, pp. 225-233, 1998.

[32] A. R. Virk, M. A. Rehman, and W. Nazeer, "New definition of atomic bond connectivity index to overcome deficiency of structure sensitivity and abruptness in existing definition," Scientific Inquiry and Review, vol. 3, no. 4, pp. 1-20, 2019.

[33] J.-B. Liu, M. Younas, M. Habib, M. Yousaf, and W. Nazeer, "M-polynomials and degree-based topological indices of $\operatorname{VC5C7}[p, q]$ and HC5C7 $[p, q]$ nanotubes," IEEE Access, vol. 7, pp. 41125-41132, 2019.

[34] Z. Shao, A. R. Virk, M. S. Javed, M. A. Rehman, and M. R. Farahani, "Degree based graph invariants for the molecular graph of Bismuth Tri-Iodide," Engineering and Applied Science Letter, vol. 2, no. 1, pp. 1-11, 2019.

[35] A. u. R. Virk, M. N. Jhangeer, M. N. Jhangeer, and M. A. Rehman, "Reverse Zagreb and reverse hyper-zagreb indices for silicon carbide $\mathrm{Si}_{2} \mathrm{C}_{3}-\mathrm{I}[r, s]$ and $\mathrm{Si}_{2} C_{3}-\mathrm{II}[r, s]$." Engineering and Applied Science Letters, vol. 1(2018), no. 2, pp. 37-50, 2018. 